P144 SPECIALIST DOMICILIARY PHARMACY INTERVENTION MAY REDUCE EXACERBATION FREQUENCY AND HOSPITALISATION IN PATIENTS WITH SEVERE CHRONIC OBSTRUCTIVE PULMONARY DISEASE

V Hunt, D Anderson, R Lowrie. Queen Elizabeth University Hospital, Glasgow, UK

10.1136/thoraxjnl-2016-209333.287

COPD is the $3^{\text {rd }}$ most common cause for hospital admission in the UK. Patients with COPD are often unable to attend specialist clinics, when factors contributing to exacerbations can be treated, due to the unstable nature of the condition and the degree of breathlessness experienced. There is also a high level of polypharmacy in this population. Domiciliary specialist pharmacy intervention may help identify and treat these issues.

Hypothesis Specialist Domiciliary Pharmacy intervention reduces exacerbation frequency and hospitalisation in patients with Severe COPD

Patients who were referred to a specialist COPD clinic between March 2015 and January 2016 were assessed. Those with polypharmacy and high exacerbation frequency or severe breathlessness (MRC grade 4-5) were identified and offered domiciliary pharmacy review. Patients who consented were visited at home with a comprehensive review of medication, the case was then discussed at an MDT level and therapeutic changes implemented. A comparator group of matched patient were identified and followed up without pharmacy intervention.

Baseline data were collected including demographics, smoking status, $\mathrm{FEV}_{1}$, MRC Grade, hospitalisation rates, exacerbation frequency and current medications. Patient records were then reviewed after a 6 month period to look at impact of intervention on hospitalisation, exacerbation frequency, antibiotic and steroid prescription in the two groups.

Results A total of 88 patients received intervention and 87 patients were followed up in a comparator group. Patients were matched in terms of sex, BMI, socioeconomic deprivation, smoking status, $\mathrm{FEV}_{1}, \mathrm{MRC}$ Grade, LTOT use and hospitalisation frequency.

Patients had an average of 3.5 visits with an average of 7.6 interventions per patient, therapeutic changes being the most frequent.
Review after 6 months of intervention demonstrated that the intervention group had a significant reduction in hospitalisation $(\mathrm{p}<0.01)$ and exacerbation frequency $(\mathrm{p}<0.01)$ (Figure 1) along with antibiotic and steroid use in comparison to the comparator group.

This project was a service evaluation rather than a double blinded, randomised control study. The results however suggest that specialist pharmacy intervention in a setting appropriate for a disabled population has a positive impact on exacerbation frequency and hospitalisation.

\section{P145 EVALUATION AND QUANTIFICATION OF TREATMENT PREFERENCES FOR PATIENTS WITH ASTHMA OR COPD USING DISCRETE CHOICE EXPERIMENT SURVEYS}

${ }^{1} \mathrm{H}$ Svedsater, ${ }^{1} \mathrm{E}$ Hilton, ${ }^{1} \mathrm{D}$ Leather, ${ }^{2} \mathrm{~T}$ Robinson, ${ }^{3} \mathrm{~L}$ Bradshaw, ${ }^{4} \mathrm{H}$ Doll, ${ }^{4} \mathrm{~B}$ Nafees. ${ }^{1}$ GlaxoSmithKline, Stockley Park, UK; ${ }^{2}$ Harrogate District Hospital, Harrogate, UK; ${ }^{3}$ Northern General Hospital, Sheffield, UK; ${ }^{4}$ ICON Clinical Research UK Ltd, Abingdon, UK

\subsection{6/thoraxjnl-2016-209333.288}

Background The impact of asthma and chronic obstructive pulmonary disease (COPD) on patients' lives can be substantial, despite the use of maintenance medication. To understand the treatment preferences of patients with asthma or COPD, a discrete choice experiment (DCE) survey was developed, based on factors identified in a previous stage of this study as being influential. ${ }^{1}$

Methods UK residents aged $\geq 18$ years currently receiving treatment for asthma or COPD were recruited by a commercial recruitment panel (Global Perspectives) to take part in an internet-based survey. Participants provided demographic information and ranked eight attributes of treatment using an 8-point Likert scale $(0=$ extremely important $-8=$ not at all important). Furthermore, treatment preferences were elicited by a DCE survey; participants chose between two hypothetical treatments that differed in terms of previously identified influential attributes. ${ }^{1} \mathrm{~A}$ mixed logit regression model assessed DCE preferences for each condition separately.

Results Data were collected from 302 participants, who generally had poorly controlled asthma (indicated by Asthma Control Test $^{\mathrm{TM}}$ scores $\leq 19 / 25$ ) or experienced a high impact of COPD

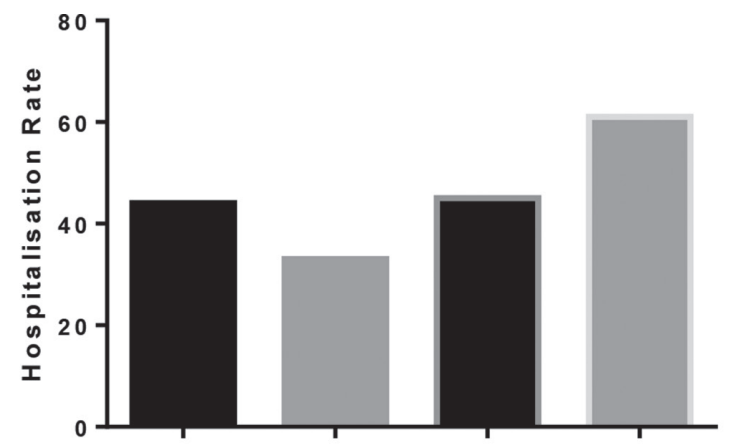

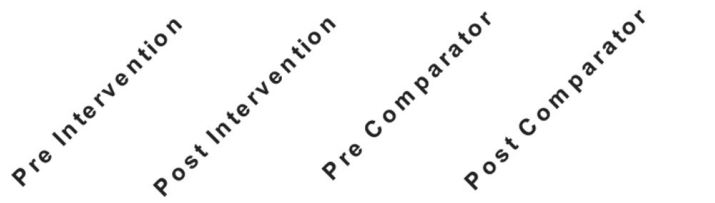

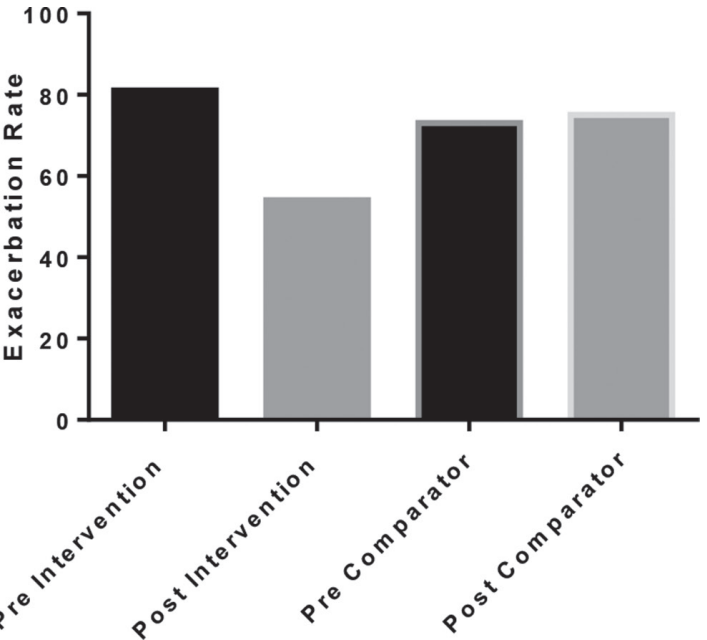

Abstract P144 Figure 1 Impact of Pharmacy Intervention on Exacerbation Rates and Hospital Admission Rates 6 months prior to and after intervention, compared to comparator group. 
Abstract P145 Table 1 Participants' characteristics, current treatment attributes and treatment preferences

\begin{tabular}{|c|c|c|}
\hline & $\begin{array}{l}\text { Asthma } \\
(\mathrm{N}=152)\end{array}$ & $\begin{array}{l}\text { COPD } \\
(\mathrm{N}=150)\end{array}$ \\
\hline \multicolumn{3}{|l|}{ Participant characteristics } \\
\hline Mean age, years (SD) & $48.2(15.5)$ & $63.7(8.23)$ \\
\hline Female gender, $\mathrm{n}(\%)$ & $87(57.2)$ & $66(44.0)$ \\
\hline Years of having asthma/COPD, mean (SD) & $23.4(14.7)$ & $8.54(5.35)$ \\
\hline Severity of asthma/COPD, mean score (SD) $)^{1}$ & $18.01(4.65)$ & $22.8(8.6)$ \\
\hline Quality of life, mean score (SD) $)^{2}$ & $5.34(1.17)$ & $55.2(21.4)$ \\
\hline Participants not paying for their treatment, $\mathrm{n}(\%)$ & $96(63.2)$ & $141(94.0)$ \\
\hline \multicolumn{3}{|c|}{ Most frequent responses for each attribute of participants' current treatment, $n(\%)$} \\
\hline Proportion of the day that symptoms were well controlled & 'Most': 64 (41.1) & 'Most': 62 (41.3) \\
\hline How many desired physical activities participants could do/day & 'Most': 80 (52.6) & 'Some': 73 (48.7) \\
\hline Sleep disturbance, nights/week participants typically woke up & 'None': 66 (43.4) & 'None': 65 (43.3) \\
\hline Flare ups/exacerbations of asthma/COPD symptoms/year & 'Two-three': 67 (44.1) & 'Two-three': 46 (30.7) \\
\hline Times/day participants needed to take maintenance medication & 'Twice': 88 (57.9) & 'Twice': 89 (59.3) \\
\hline Ease of use and convenience of inhaler & 'V. easy': 115 (75.7) & 'V. easy': 106 (70.7) \\
\hline How many desired social activities participants could do/day & 'All': 82 (53.9) & 'Most': 53 (35.3) \\
\hline Cost/month of asthma/COPD medication ${ }^{3}$ & '<f10': $36(64.3 \%)$ & 'sf10': $6(66.7 \%)$ \\
\hline \multicolumn{3}{|l|}{ Ranking of importance of treatment attributes, mean (SD) ${ }^{4}$} \\
\hline Proportion of the day that symptoms were well controlled & $3.27(1.93)$ & $3.11(1.92)$ \\
\hline How many desired physical activities participants could do/day & $3.98(2.04)$ & $3.82(1.70)$ \\
\hline Sleep disturbance, nights/week participants typically woke up & $4.27(2.08)$ & $4.53(2.10)$ \\
\hline Flare ups/exacerbations of asthma/COPD symptoms/year & $4.11(1.90)$ & $3.79(1.86)$ \\
\hline Times/day participants needed to take maintenance medication & $4.51(2.48)$ & $4.15(2.53)$ \\
\hline Ease of use and convenience of inhaler & $4.69(2.23)$ & $4.87(2.16)$ \\
\hline How many desired social activities participants could do/day & $5.11(1.96)$ & $4.59(1.84)$ \\
\hline Cost/month of asthma/COPD medication & $6.06(2.58)$ & $7.14(1.78)$ \\
\hline \multicolumn{3}{|l|}{ Preferences for treatment, $\mathrm{OR}(95 \% \mathrm{Cl})$} \\
\hline Not waking up vs waking up $3-4$ times/week & $3.02(2.71-3.37)^{5}$ & $2.84(2.52-3.20)^{5}$ \\
\hline Costs no more than $f 10$ vs $f 50 /$ month & $2.90(2.60-3.24)^{5}$ & $3.95(3.50-4.47)^{5}$ \\
\hline V. easy and convenient to use vs fairly difficult and inconvenient & $1.91(1.72-2.12)^{5}$ & $1.95(1.74-2.18)^{5}$ \\
\hline Experience flare ups/exacerbations no vs two-three times/year & $1.69(1.52-1.88)^{5}$ & $2.43(2.17-2.73)^{5}$ \\
\hline Able to do all vs some desired physical activities/day & $1.57(1.41-1.74)^{5}$ & $1.60(1.44-1.79)^{5}$ \\
\hline Symptoms are stable and well controlled all vs some of the day & $1.48(1.34-1.65)^{5}$ & $1.64(1.47-1.84)^{5}$ \\
\hline Able to do all vs some desired social activities/day & $1.26(1.13-1.40)^{5}$ & $1.39(1.25-1.56)^{5}$ \\
\hline Need to take medication once a day vs three times/day & $1.14(1.03-1.27)^{6}$ & $1.15(1.03-1.29)^{6}$ \\
\hline
\end{tabular}

${ }^{1}$ Total score of ACT (asthma)/CAT (COPD). ${ }^{2}$ Total score of the AQLQ-S for patients with asthma or the SGRQ-C. ${ }^{3}$ For participants who were paying for their medication. ${ }^{4}$ Treatment attributes were ranked using an 8-point Likert scale $\left(0=\right.$ extremely important- $8=$ not at all important). ${ }^{5} \mathrm{p}<0.001$. ${ }^{6} \mathrm{p}=0.016$. ACT, asthma control test; AQLQ-S, standardised asthma quality of life questionnaire; CAT, COPD assessment test; Cl, confidence interval; OR, odds ratio; SD, standard deviation; SGRQ-C, St George's respiratory questionnaire for patients with COPD; V, very.

(indicated by COPD Assessment Test ${ }^{\mathrm{TM}}$ scores $>20 / 40$ ) and had been living with asthma or COPD for approximately 23.4/8.5 years (Table). Most attributes of participants' current treatment were similar for the asthma and COPD populations; participants ranked having well-controlled symptoms all day as most important while the monthly cost was ranked as least important (Table). Treatment preferences from most to least important (with some degree of variation between conditions) were: reduced sleep disturbance, inexpensive, easy/convenient to use, reduced flare ups/ exacerbations, enabled participation in all desired physical activities, controlled symptoms all day, enabled participation in all desired social activities and reduced medication frequency (Table); all attributes were significant in influencing treatment choices.

Conclusions All identified valued attributes of medications had a significant impact in the DCE, despite differences in relative importance, and should be considered by healthcare professionals when discussing maintenance treatment options with patients with asthma and COPD. These factors should be considered in the development of future medications.

\section{REFERENCE}

1 Svedsater, et al. AJRCCM 2016;A1747.

\section{P146 MORTALITY AND DAY OF ADMISSION FOR ACUTE EXACERBATION OF COPD}

A Duffy, I Steer, SC Bourke, C Echevarria. Northumbria Healthcare NHS Foundation Trust, North Shields, UK

\subsection{6/thoraxjnl-2016-209333.289}

Background Excess mortality in patients admitted to hospital at weekends has been reported in many healthcare systems, influencing healthcare policy. The limitations of existing data are well described; there is a need for condition-specific research in welldescribed populations with adjustment for baseline mortality risk. ${ }^{1}$ Acute exacerbations of COPD (AECOPD) are one of the commonest reasons for hospital admission, with high rates of inpatient mortality. We aimed to establish if inpatient mortality is associated with day of admission or death amongst patients admitted with an AECOPD. 